Article

\title{
Laser-Plasma Spatiotemporal Cyanide Spectroscopy and Applications
}

\author{
Christian G. Parigger ${ }^{1, *(1)}$, Christopher M. Helstern ${ }^{1}$, Benjamin S. Jordan ${ }^{2}$, \\ David M. Surmick $^{3(\mathbb{1})}$ and Robert Splinter ${ }^{4}(\mathbb{D}$ \\ 1 Physics and Astronomy Department, University of Tennessee, University of Tennessee Space Institute, \\ Center for Laser Applications, 411 B.H. Goethert Parkway, Tullahoma, TN 37388, USA; \\ chris.helstern@gmail.com \\ 2 Nuclear Engineering Department, University of Tennessee, Tickle College of Engineering, \\ 1412 Circle Drive, Knoxville, TN 37912, USA; bjordan1@vols.utk.edu \\ 3 Physics and Applied Physics Department, University of Massachusetts Lowell, Lowell, MA 01854, USA; \\ David_Surmick@uml.edu \\ 4 Wellinq Medical, Van der Waals Park 22, 9351 VC Leek, The Netherlands; rsplinter@gmail.com \\ * Correspondence: cparigge@tennessee.edu; Tel.: +1-(931)-841-5690
}

Received: 13 January 2020; Accepted: 28 January 2020; Published: 31 January 2020

\begin{abstract}
This article reports new measurements of laser-induced plasma hypersonic expansion measurements of diatomic molecular cyanide (CN). Focused, high-peak-power $1064 \mathrm{~nm}$ Q-switched radiation of the order of $1 \mathrm{TW} / \mathrm{cm}^{2}$ generated optical breakdown plasma in a cell containing a 1:1 molar gas mixture of $\mathrm{N}_{2}$ and $\mathrm{CO}_{2}$ at a fixed pressure of $1.1 \times 10^{5} \mathrm{Pascal}$ and in a $100 \mathrm{~mL} / \mathrm{min}$ flow of the mixture. Line-of-sight (LOS) analysis of recorded molecular spectra indicated the outgoing shockwave at expansion speeds well in excess of Mach 5. Spectra of atomic carbon confirmed increased electron density near the shockwave, and, equally, molecular $\mathrm{CN}$ spectra revealed higher excitation temperature near the shockwave. Results were consistent with corresponding high-speed shadowgraphs obtained by visualization with an effective shutter speed of 5 nanoseconds. In addition, LOS analysis and the application of integral inversion techniques allow inferences about the spatiotemporal plasma distribution.
\end{abstract}

Keywords: plasma diagnostics; molecular spectra; diatomic molecules; plasma spectroscopy; laser spectroscopy; laser-induced breakdown spectroscopy; optical emission spectroscopy; hypersonic expansion

\section{Introduction}

Molecular-recombination spectra are readily measured following optical breakdown in gases. The cyanide $(\mathrm{CN})$ molecule can be recognized rather early for time delays of the order of $100 \mathrm{~ns}$ after cessation of the laser pulse [1]. Gas mixtures are selected for the purpose of measuring CN spatiotemporal distributions associated with high-speed, hypersonic gas-dynamic expansion. The main goals and objectives of this work comprise (a) exploring CN measurement using optical emission spectroscopy subsequent to optical breakdown; (b) determining the effects of laser-induced shockwave for time delays of the order of $1 \mu \mathrm{s}$; (c) evaluating the spatial distribution of $\mathrm{CN}$ signals; (d) inferring $\mathrm{CN}$ temperature distribution; and (e) associating recorded shadowgraphs with laser-spectroscopy results. Applications of cyanide spectroscopy include various scientific areas, including medical research and selected engineering endeavors. In this work, formation of $\mathrm{CN}$ molecules occurs through recombination subsequent to laser-induced optical breakdown. Laser plasma is studied by measuring spatial distribution along the optical axis for selected time delays. For the mixture and flowing-gas 
experiments, chemical composition was governed by free but shockwave-affected expansion associated with laser-induced plasmas in gases.

Cyanides are connected to many living organisms, and are created through artificial processes, but they are highly toxic. Human exposure can occur through accidental ingestion or inhalation of cyanides. Synthetic materials can be found in 21st century buildings and furnishings that produce toxic-combustion byproducts like hydrogen cyanide ( $\mathrm{HCN})$ when burned [2]. Victims of these fires can have significant intake of $\mathrm{HCN}$, which can lead to cyanide poisoning and ultimately death. Cyanide detection can identify cyanide-poisoning occurrences in humans and can assist in the determination of adequate treatment. Additionally, $\mathrm{HCN}$ can be produced by bacteria such as Pseudomonas aeruginosa [3]. Immune-compromised patients, such as those with cystic fibrosis (CF), are susceptible to increased morbidity and mortality when afflicted by these bacteria [3]. Detection of bacterial-produced $\mathrm{HCN}$ in the airways leads to earlier treatment of immune-compromised infected with these bacteria.

Diatomic molecule $\mathrm{CN}$ has remained a target for improved detection capability and deeper understanding, largely because engineers cannot replicate its effectiveness for certain industrial applications with any other compound [4]. For example, $90 \%$ of global gold-mining operations utilize $\mathrm{CN}$ because of its availability, effectiveness, and cost efficiency [5]. Although accidents, injuries, and environmental mishaps are possible, when they occur, they require specialized detection capability to ensure worker safety during remediation.

Engineering applications associated with plastic manufacturing that continue to use cyanide create a downstream problem for firefighters, analogous to that of fire victims mentioned above. Firefighters must go into buildings where plastics that may have used cyanide during their manufacture then evolve their constituents into the air as they are consumed by fire [6]. Again, detection and assessment of the amount of $\mathrm{CN}$ present is needed to help keep firefighters safe.

Engineers continue to look for means of obtaining $\mathrm{CN}$ properties that customers need but that do not have the potential health hazards that are present with $\mathrm{CN}[7,8]$. Engineers have been seeking ways to lower cyanide utilization in the nitrile chemical industry [9]. Alternatives to cyanide are also sought for the mining industry, but this task has proven difficult because replacement candidates are often not as broadly applicable and must be tailored to the specific ore [10].

In addition, $\mathrm{CN}$ is a component of vehicle exhaust [11-15]. It is important for engineers to continue to research the details of $\mathrm{CN}$ detection and evaluation to be able to characterize the relative emission impact that different vehicles contribute [16]. There is $\mathrm{CN}$ production potential in other combustion processes as well, including the CN spectroscopy of trinitrotoluene (TNT) simulant molecules [17], and continued understanding of what level of $\mathrm{CN}$ production should be expected continues to drive interest and investigation into $\mathrm{CN}$, even if engineers were able to replace $\mathrm{CN}$ in many industrial applications [18].

\section{Experiment Details}

The standard experiment components were used for laser-induced breakdown spectroscopy, and were summarized previously, e.g., see [1], but were included for completeness. The experiment arrangement consisted of a set of typical components for time-resolved laser-induced optical emission spectroscopy, or nanosecond laser-induced breakdown spectroscopy (LIBS) [19]. Primary instrumentation includes a Q-switched neodymium-doped yttrium aluminum garnet, $\mathrm{Nd}: \mathrm{Y}_{3} \mathrm{Al}_{5} \mathrm{O}_{1} 2$ (Nd:YAG) device (Quantel model Q-smart 850, USA) operated at the fundamental wavelength of 1064 $\mathrm{nm}$ to produce full-width-at-half-maximum $6 \mathrm{~ns}$ laser radiation with an energy of $850 \mathrm{~mJ}$ per pulse, a laboratory-type Czerny-Turner spectrometer (Jobin Yvon model HR 640, Fr) with $0.64 \mathrm{~m}$ focal length, equipped with a 1200 groove/mm grating, an intensified charge coupled device (Andor Technology model iStar DH334T-25U-03, USA) for recording temporally and spatially resolved spectral data, a laboratory chamber or cell with inlet and outlet ports, and a vacuum system, electronic components for synchronization, and various optical elements for beam shaping, steering, and focusing.

For the generation of optical-breakdown microplasma, a singlet lens (Thorlabs model LA1509-C, USA) was used close to the top entrance window of one arm of the chamber containing 
the 1:1 $\mathrm{CO}_{2}: \mathrm{N}_{2}$ atmospheric molar gas mixture (Airgas ultrahigh-purity $\mathrm{N}_{2}$ and research-grade $\mathrm{CO}_{2}$ ). For 1:1 imaging of the plasma onto the $100 \mu \mathrm{m}$ spectrometer slit, a fused silica planoconvex lens (Thorlabs model LA4545, USA) was employed. For the CN experiments reported here, laser-pulse energy was attenuated with beamsplitters and apertures from 850 to $170 \mathrm{~mJ} /$ pulse. Measurements were performed with and without an Order Sorting Filter (Oriel model 51250, USA), with a cut-on wavelength of $309 \mathrm{~nm}$ and transmittance range of $325 \mathrm{~nm}$, to evaluate the CI $193.09 \mathrm{~nm}$ atomic carbon line in second-order interference as identified in previous work [1].

In previous experiments, captured shadowgraphs of the breakdown plasma [20] served the purpose of visualizing the plasma expansion when using $850 \mathrm{~mJ}, 6 \mathrm{~ns}$ radiation. However, it is important to obtain shadowgraphs for plasma-excitation energies that were employed for time-resolved spectroscopy. Shadowgraphs reported in this work were captured using two separate laser devices (Continuum Surelite model SL I-10, USA) that could be externally operated to deliver laser pulses with a well-defined time delay showing less than $\pm 1 \mathrm{~ns}$ trigger-jitter between the pulses. For visualization studies, both lasers were frequency-doubled to operate at the 2 nd harmonic, $532 \mathrm{~nm}$ wavelength, and both beams were spatially overlapped. Both pulses could be delivered with a minimal time delay of $300 \mathrm{~ns}$. Shadowgraphs were recorded by external synchronization of the Surelite and Quantel laser devices, and by externally triggering the camera (Silicon Video 9C10 color camera, USA) that recorded the images that were projected onto a screen.

\section{Results and Discussion}

\subsection{Shadowgraphs}

Two typical images are reported here. First, Figure 1 displays a shadowgraph of the IR $1064 \mathrm{~nm}$ laser-induced plasma for an excitation energy of $170 \mathrm{~mJ} 6 \mathrm{~ns}$ pulse width. The shadowgraph was captured using a single $5 \mathrm{~ns}$ pulse-width $532 \mathrm{~nm}$ beam.

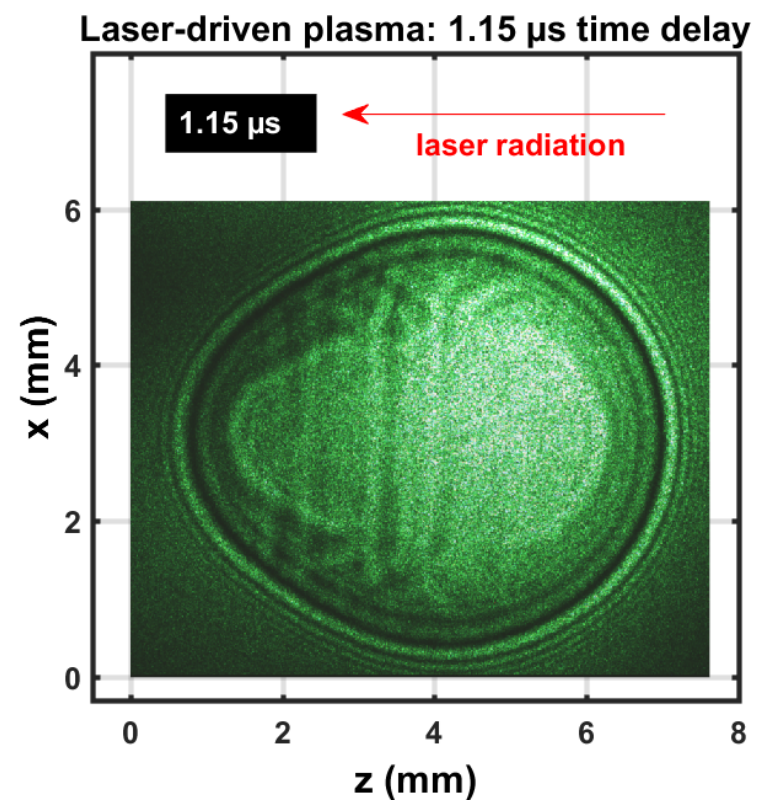

Figure 1. Single-shot shadowgraph of expanding laser-induced plasma initiated with a $170 \mathrm{~mJ}$, 6'ns, $1064 \mathrm{~nm}$ focused beam, and imaged using a $5 \mathrm{~ns}, 532 \mathrm{~nm}$ backlight that was time-delayed by $1.15 \pm 0.05 \mu \mathrm{s}$.

The expanding shockwave was clearly visible, including the central plasma kernel. The image was nearly spherical; previously reported shadowgraphs that utilized $850 \mathrm{~mJ}$ excitation would reveal stronger deviation from spherical symmetry than the images obtained with a $170 \mathrm{~mJ} / \mathrm{pulse}$. 
The expanding shockwave radius was consistent with previous assessments of hypersonic laser-plasma expansion images [20] that employed Taylor-Sedov energy $y^{1 / 5}$ dependency [21],

$$
R(\tau)=\left(\frac{E}{\rho} \tau^{2}\right)^{1 / 5}
$$

of shockwave radius $R(\tau)$ as function of absorbed pulse energy $E$, gas density $\rho$, and time delay $\tau$. Tables 1 and 2 compare for energies of 160 and $200 \mathrm{~mJ}$, respectively, computed blast-wave radii for standard ambient temperature and pressure (SATP) air, and the CN mixture. Consequently, measured shadowgraphs in air provided an excellent guide for the gaseous mixture.

Table 1. Computed shockwave radii for standard ambient temperature and pressure (SATP) air and for molar cyanide $(\mathrm{CN})$ mixture, $160 \mathrm{~mJ}$.

\begin{tabular}{ccc}
\hline Time Delay $(\mathbf{n s})$ & $\mathbf{r}(\mathbf{m m})$ for Air $\left(\rho=\mathbf{1 . 2} \mathbf{~ k g} / \mathbf{m}^{\mathbf{3}}\right)$ & $R(\mathbf{m m})$ for CN Mix $\left(\rho=\mathbf{1 . 6 3} \mathbf{~ k g} / \mathbf{m}^{\mathbf{3}}\right)$ \\
\hline 200 & 1.40 & 1.31 \\
450 & 1.93 & 1.82 \\
700 & 2.31 & 2.17 \\
950 & 2.61 & 2.45 \\
1200 & 2.86 & 2.69 \\
1450 & 3.09 & 2.90 \\
\hline
\end{tabular}

Table 2. Computed shockwave radii for SATP air and molar CN mixture, $200 \mathrm{~mJ}$.

\begin{tabular}{ccc}
\hline Time Delay $(\mathbf{n s})$ & $\mathbf{r}(\mathbf{m m})$ for Air $\left(\rho=\mathbf{1 . 2} \mathbf{~ k g} / \mathbf{m}^{\mathbf{3}}\right)$ & $R(\mathbf{m m})$ for CN Mix $\left(\rho=\mathbf{1 . 6 3} \mathbf{~ k g} / \mathbf{m}^{\mathbf{3}}\right)$ \\
\hline 200 & 1.46 & 1.37 \\
450 & 2.02 & 1.90 \\
700 & 2.41 & 2.27 \\
950 & 2.73 & 2.56 \\
1200 & 2.99 & 2.81 \\
1450 & 3.23 & 3.04 \\
\hline
\end{tabular}

Figure 2 exhibits a composite shadowgraph obtained by initiating laser plasma with the first, green, $90 \mathrm{~mJ} /$ pulse, $5 \mathrm{~ns}$ beam. Subsequently, initiating laser plasma at the shockwave with the second, green, $110 \mathrm{~mJ} /$ pulse, 5 ns beam spatially overlapped exactly, but was time-delayed by $0.45 \mu \mathrm{s}$. The composite image was obtained with the $0.45 \mu$ s time delay and an extra $30 \mathrm{~ns}$ optical time delay for each beam. The initial, elongated multiple breakdown in the central region was imaged with the first 5 ns pulse-width beam. The near-spherical shockwave was imaged with the second 5 ns pulse-width beam and the corresponding $30 \mathrm{~ns}$ time delayed beam. However, the second beam initiated breakdown at the forward side (left in image) and at the side of the incoming beam (right in image), indicated by a dark ring. The extent of that dark ring was due to the use of a 5 ns pulse-width beam, time-delayed by $30 \mathrm{~ns}$ for shadowgraph capture of expansion dynamics. The expansion speed of the second optical breakdown at the right of the image was well over one order of magnitude larger than hypersonic speed in SATP air.

Subsidiary measurement utilized a pyroelectric laser-pulse energy meter (Scientech model P50, USA) for determination of absorbed energy for the laser-induced plasma studies. Transmitted energy per pulse $E_{t}$ amounted to $20 \pm 3 \mathrm{~mJ}$ for IR laser-induced plasma studies. This energy/pulse represents the optical-breakdown threshold. The energy/pulse threshold corresponded to the peak-power per area or irradiance threshold for SATP air optical breakdown [22]. For example, for perfect Gaussian beam focusing $[23,24]$ with $\mathrm{f} / 20$, i.e., $F / D=20$ with $F$ and $D$ denoting the focal length and the beam diameter at the lens, respectively, for $1064 \mathrm{~nm}$ IR wavelength, $\lambda$, and from the beam radius, $w_{0}$,

$$
w_{0}=\frac{2}{\pi} \lambda \frac{F}{D}
$$


one finds the focal area of $5.8 \times 10^{-6} \mathrm{~cm}^{2}$. Using for $\Delta \tau_{\text {pulse }}=6 \mathrm{~ns}$ pulses the peak-power, $\frac{1}{2} E_{t} / \Delta \tau_{\text {pulse' }}$ of 1.7 MW (mean-field top-hat model for the temporal pulse distribution) one would find the irradiance threshold of $2.9 \times 10^{11} \mathrm{GW} / \mathrm{cm}^{2}$. In view of Equation (1) for determination of shockwave radius, the absorbed energy per pulse should be used. However, the 1/5 exponent implied diminished dependency on the energy/pulse when in the range of 160 and $200 \mathrm{~mJ}$, as displayed in Tables 1 and 2. In other words, when considering effects from 220 and $200 \mathrm{~mJ}$ per pulse laser-plasma generations, a ratio of $220 / 200=1.1$ or a $10 \%$ difference in energy/pulse would result in a $2 \%$ difference in the predicted shockwave radius for the same density and time delay.

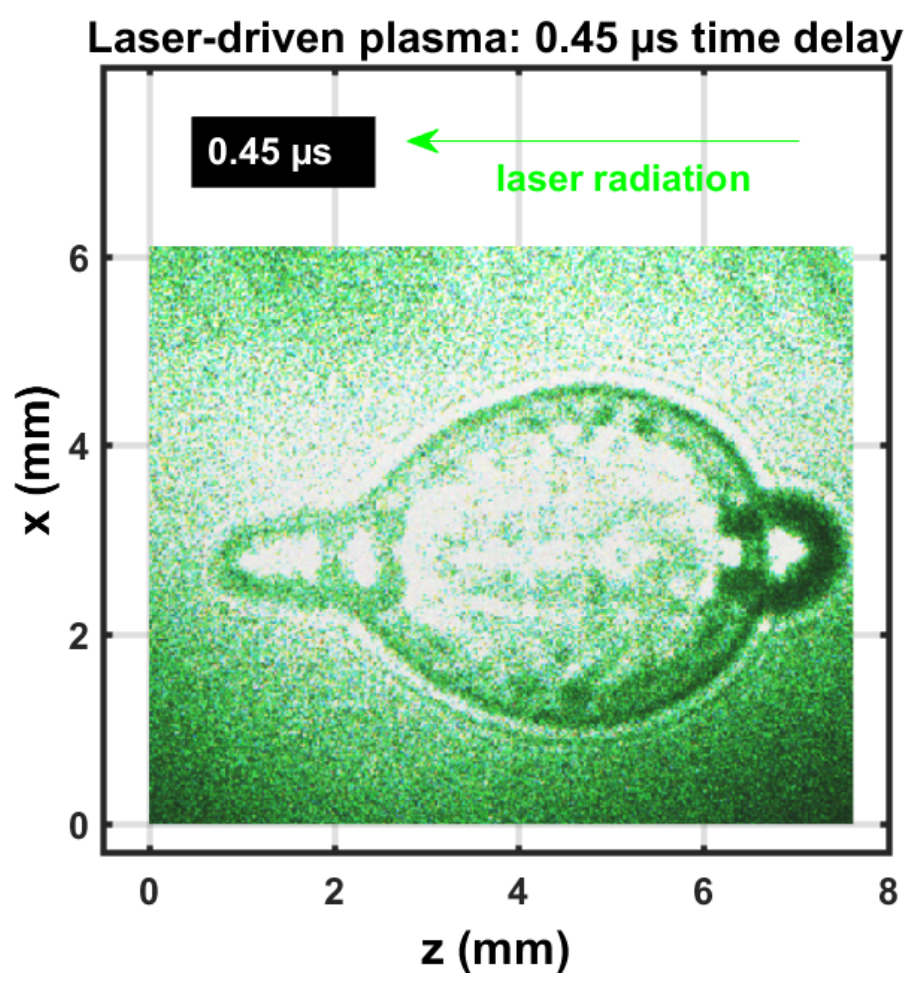

Figure 2. Single-shot shadowgraph of laser-induced plasma initiated with 90 and $110 \mathrm{~mJ}, 5 \mathrm{~ns}, 532 \mathrm{~nm}$ focused beams, and imaged using two $5 \mathrm{~ns}, 532 \mathrm{~nm}$ back lights that were time-delayed by $0.03,0.45$, and $0.48 \mu \mathrm{s}$.

\subsection{Cyanide Spectra}

The experiment series for the measurement of $\mathrm{CN}$ molecular distribution after optical breakdown includes evacuating the cell to a nominal backing pump pressure of the order of $1 \mathrm{~Pa}\left(10^{-2} \mathrm{Torr}\right)$, followed by generating the mixture from ultrahigh pure $\mathrm{N}_{2}$ and research-grade $\mathrm{CO}_{2}$. Optical breakdown was generated inside the chamber at a rate of $10 \mathrm{~Hz}$, with the laser beam focused with $\mathrm{f} / 5$ optics from the top, or parallel to the slit. The detector pixels were binned in four-pixel tracks along the slit direction, resulting in obtaining 256 spectra for each time delay. Recording of measurements with and without the Order-Sorting filter consist of 100 accumulations collected for 21 time delays at $250 \mathrm{~ns}$ steps. These runs allowed for the evaluation of CN plasma spatial and temporal characteristics, and the C I $193.09 \mathrm{~nm}$ atomic carbon line in second order. Figures 3 and 4 illustrate spatiotemporal spectra that were recorded along the line of sight and were accumulated over 100 individual laser-plasma events. The slit height corresponds to the z-direction, and the line of sight corresponds to the $y$-direction in reference to Figures 1 and 2. 
(a)

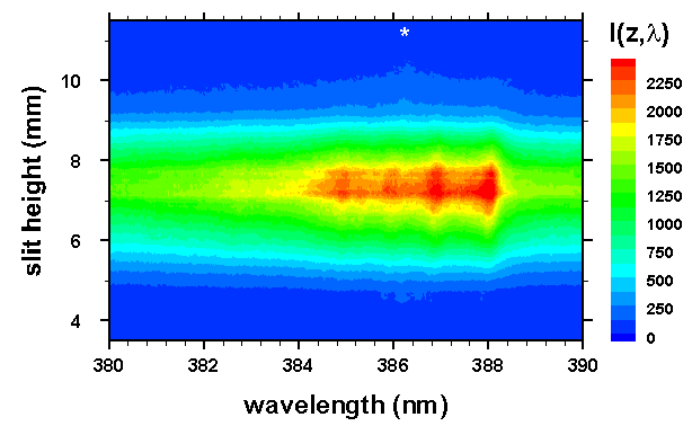

(c)

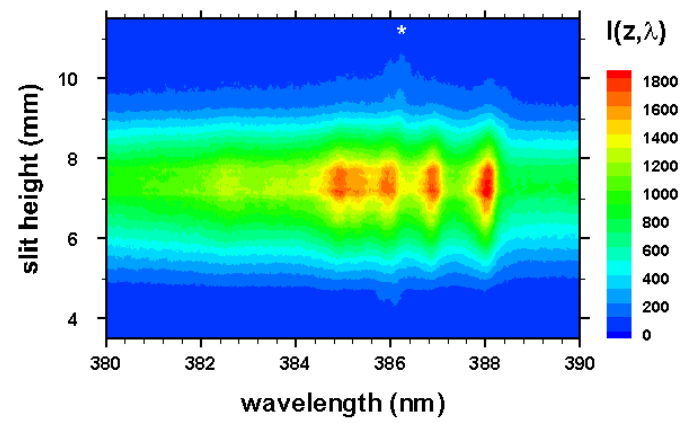

(b)

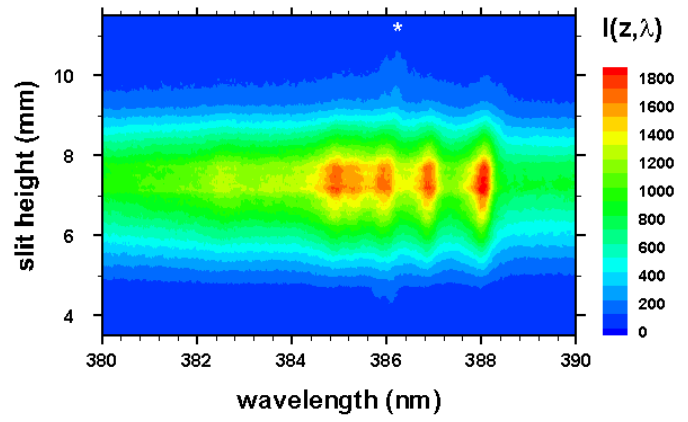

(d)

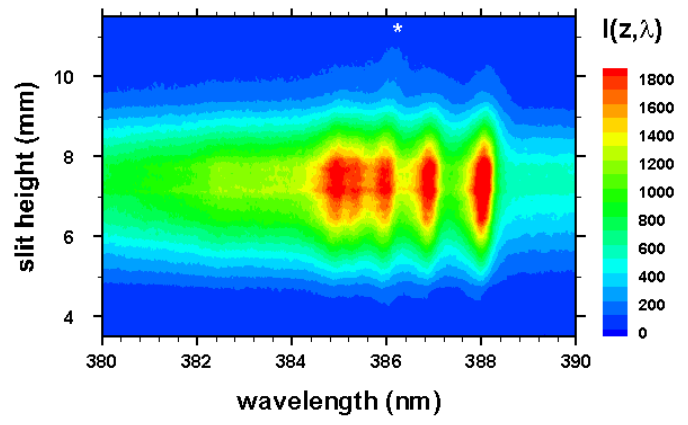

Figure 3. Optical breakdown $\mathrm{CN}$ spectra in a 1:1 molar $\mathrm{CO}_{2}: \mathrm{N}_{2}$ atmospheric gas mixture for time delays of (a) 200, (b) 450, (c) 700, and (d) 950 ns. Spectrometer-detector gate width: $125 \mathrm{~ns}$. ${ }^{*}$, second-order neutral carbon line.

(a)

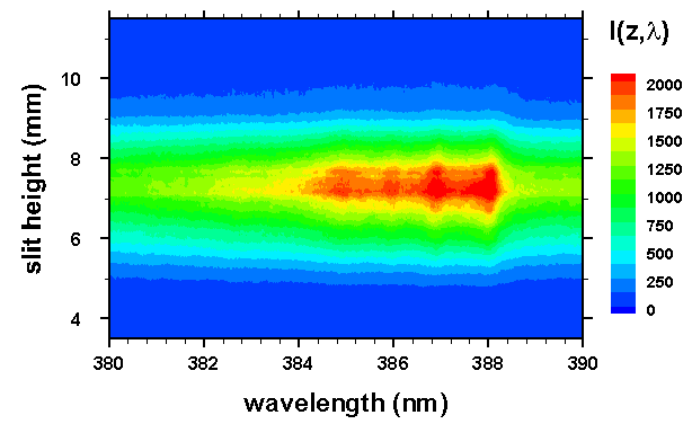

(c)

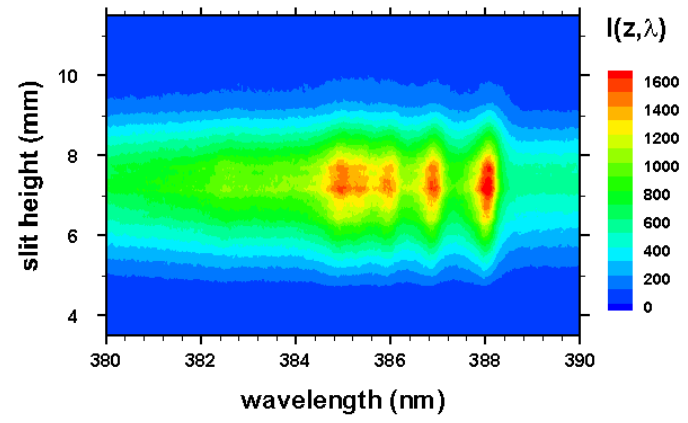

(b)

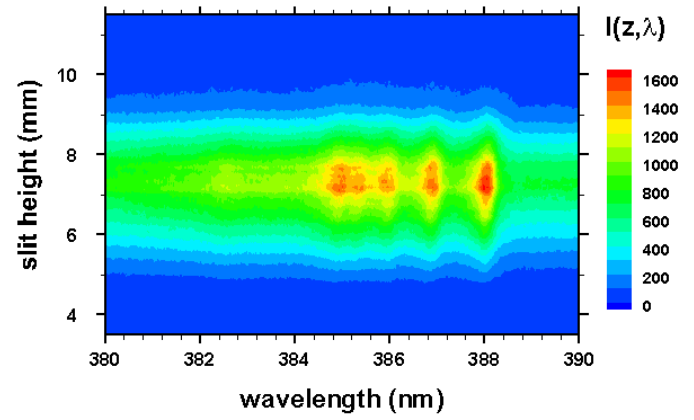

(d)

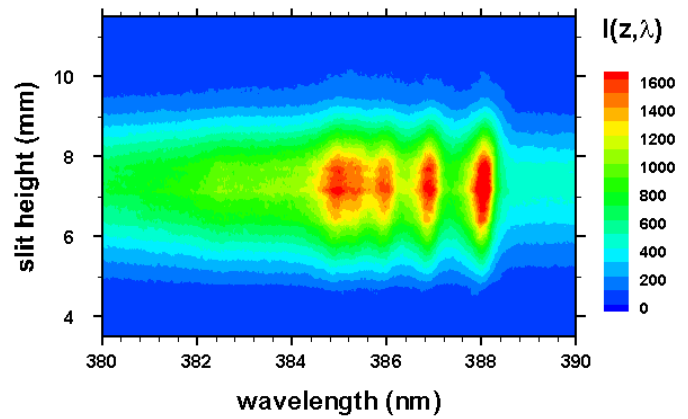

Figure 4. Optical breakdown CN spectra as in Figure 3 for time delays of (a) 200, (b) 450, (c) 700, and (d) $950 \mathrm{~ns}$, recorded with $309 \mathrm{~nm}$ cut-on wavelength filter for suppression of $193.09 \mathrm{~nm}$ neutral carbon line contributions in second order. 


\subsection{Cyanide Temperature}

Both line-of-sight and Abel-inverted datasets of the $\mathrm{CN}$ spectra were subjected to analysis with the Nelder-Mead temperature (NMT) program that uses the Nelder-Mead algorithm [25] and was made available along with the required $\mathrm{CN}$ violet system dataset $[26,27]$. The fitting utilized the NMT program and CN line-strength data; both are available in [26]. The theoretical background and development of the line-strength data are found in [27]. Figure 5 illustrates a typical CN spectrum and fit of line-of-sight data accomplished in this work.

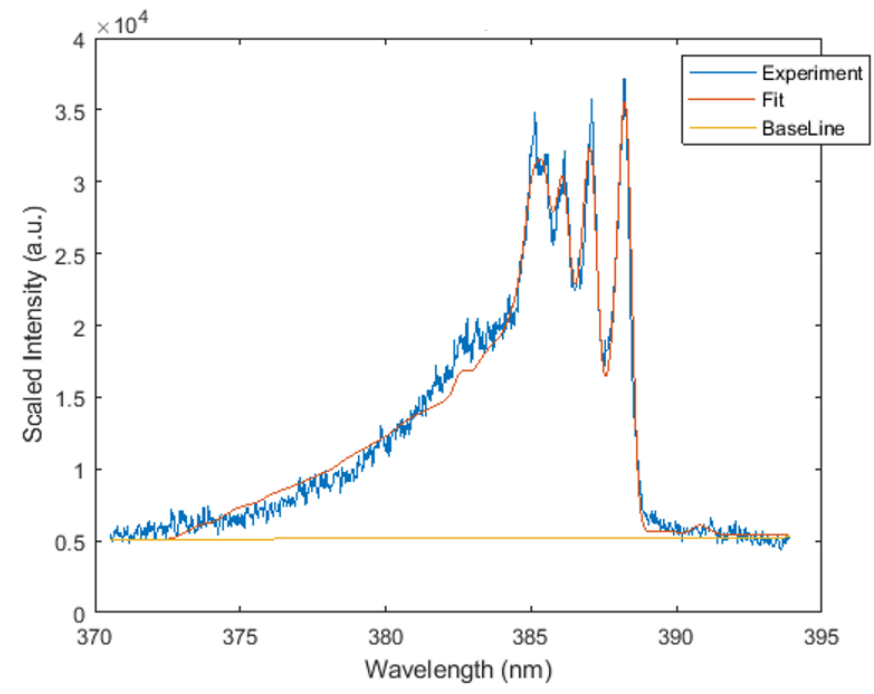

Figure 5. Typically fitted CN spectrum, $T=8947 \mathrm{~K}$, fit $F W H M=0.47 \mathrm{~nm}$.

Figure 6 exhibits analysis results of the filtered, line-of-sight molecular $\mathrm{CN}$ spectra. The figures reveal the occurrence of the outgoing shockwave, along with temperature variations in the central region of the plasma. These data of slit height vs. temperature show increased temperature near the edges.
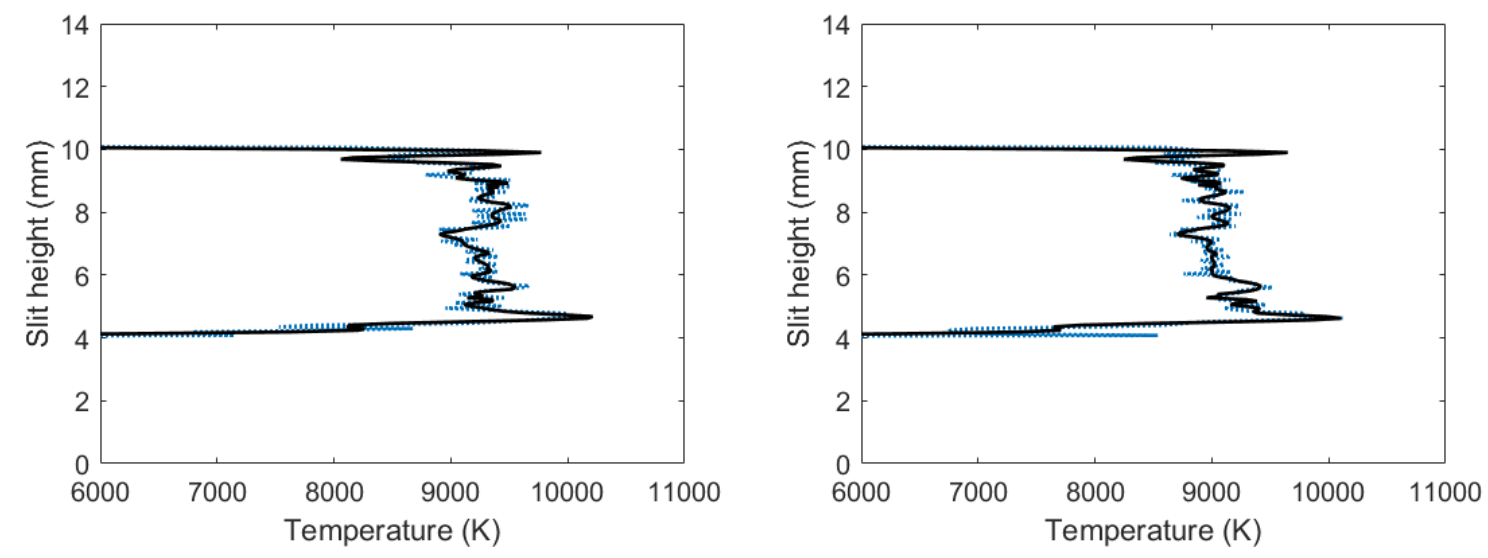

Figure 6. Temperature vs. slit height for filtered CN spectra with (left) 700 and (right) 950 ns time delay.

\subsection{Electron Density}

Unfiltered spectra contained an overlap of the C I $193.09 \mathrm{~nm}$ atomic carbon line in second order and the 2-2 CN band head of $386.19 \mathrm{~nm}$, where filtered spectra only contained the 2-2 CN band head of $386.19 \mathrm{~nm}$. C I $193.09 \mathrm{~nm}$ atomic carbon line in second-order Stark widths were evaluated for filtered and unfiltered spectra using peak-fitting programs. The difference between the filtered and unfiltered spectra was obtained by applying deconvolution. Figure 7 illustrates the results for the widths at full 
width half maximum of the carbon peak. Figure 7 reveals that larger Stark widths were seen near the edges of the plasma, while smaller Stark widths were near the center of the plasma.
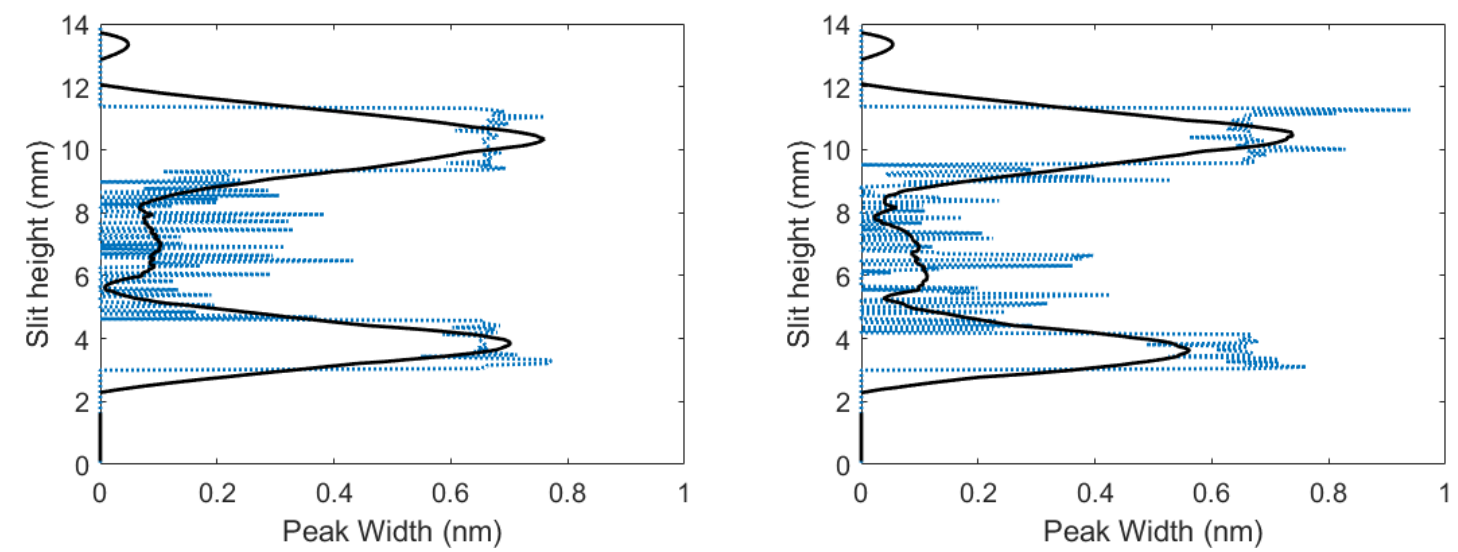

Figure 7. Inferred widths of C I $193.09 \mathrm{~nm}$ atomic carbon line in second order vs. slit height for time delays of (left) 700 and (right) $950 \mathrm{ns.}$

Electron number density $n_{e}$ can be determined from the Stark full width at half maximum, $\Delta \lambda_{\text {Stark, }}$ of the C I193.09 $\mathrm{nm}$ atomic carbon line [28], measured in second order,

$$
\Delta \lambda_{\text {Stark }}(\mathrm{nm})=2 w(\mathrm{~nm}) n_{e}\left(10^{17} \mathrm{~cm}^{-3}\right)
$$

where width parameter, $w$ was extrapolated $[28,29]$ to amount to $w \approx 0.0029 \mathrm{~nm}$. Figure 8 displays the calculated electron densities versus slit height. The calculated electron densities are of the order of $n_{e} \simeq 10^{17} \mathrm{~cm}^{-3}$ in the central region. Higher electron densities were seen near the edges of the plasma, while smaller electron densities were near the center of the plasma.

Using the Taylor-Sedov blast-wave model (see Equation (1)), plasma radius $R_{\text {plasma }}$, at time delay of $450 \mathrm{~ns}$, was $\approx 1.9 \mathrm{~mm}$, which would indicate higher electron density near the shockwave, which is consistent with results shown in Figure 8.
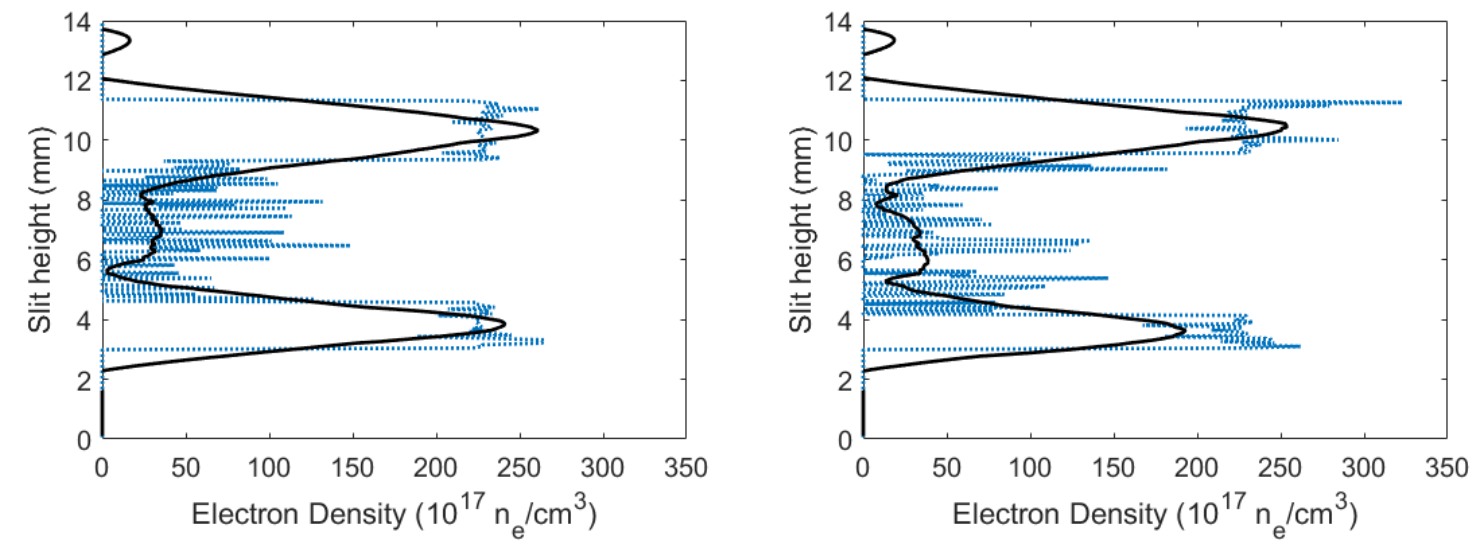

Figure 8. Calculated electron densities versus slit height for time delays of (left) 450 and (right) 950 ns.

\subsection{Cyanide Spectra in Flowing Gas}

The experiment series for the measurement of $\mathrm{CN}$ molecular distribution after optical breakdown for the flowing-gas mixture utilized a similar process as discussed in the previous sections. The experiments included supplying the cell with a constant flow of ultrahigh pure $\mathrm{N}_{2}$ and research-grade $\mathrm{CO}_{2}$ gas mixture instead of a fixed volume of the mixture. The flow rate of the mixture entering and leaving the cell was $100 \mathrm{~mL} / \mathrm{min}$ and was monitored using a flowmeter (Cole-Parmer Instr. Co. FM 112-02ST, USA). 
Optical breakdown was generated inside the chamber at a rate of $10 \mathrm{~Hz}$, with the laser beam focused with $\mathrm{f} / 5$ optics from the top or parallel to the slit. The detector pixels were binned in four-pixel tracks along the slit direction, resulting in obtaining 256 spectra for each time delay. Recording of measurements with and without the Order-Sorting filter consist of 100 accumulations collected for 21 delays at $250 \mathrm{~ns}$ steps.

Applying the NMT program to the filtered, line-of-sight molecular CN-spectra produced in the flowing-gas mixture yielded the results shown in Figure 9. The figure reveals the occurrence of the outgoing shockwave along with temperature variations in the central region of the plasma, and these slight-height vs. temperature data showed increased temperatures near the edges, analogous to the results with the fixed amount of gas mixture. Comparing these results to Figure 7 , calculated $\mathrm{CN}$ temperatures for a fixed amount of gas mixture, it appears that the highest calculated CN temperatures for Figure 7 were towards the bottom of the plasma, where the highest calculated CN temperatures for the flowing gas were towards the top of the plasma.
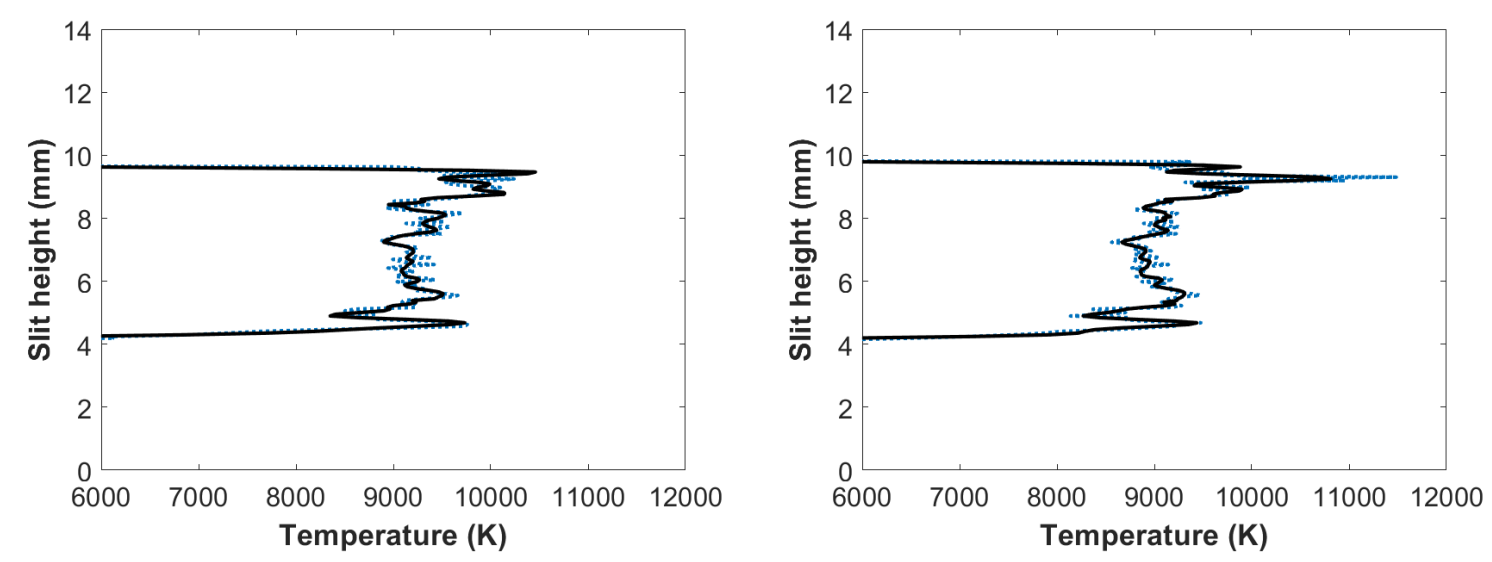

Figure 9. Temperature vs. slit height for flowing gas filtered CN spectra: (left) 700 and (right) 950 ns time delay.

Just as in the fixed gas mixture, unfiltered spectra contained an overlap of the CI193.09 $\mathrm{nm}$ atomic carbon line in second order and the 2-2 CN band head of $386.19 \mathrm{~nm}$, where filtered spectra only contained the 2-2 CN band head of $386.19 \mathrm{~nm}$. The C I $193.09 \mathrm{~nm}$ atomic carbon line in second-order Stark widths was evaluated for filtered and unfiltered spectra using peak-fitting programs, and the difference between filtered and unfiltered spectra was obtained by applying deconvolution. Figure 10 illustrates the results for the width at full-width-half-maximum of the carbon peak, and reveal that larger Stark widths were seen near the edges of the plasma, while smaller Stark width were near the center of the plasma, similar to the results in the fixed gas mixture.
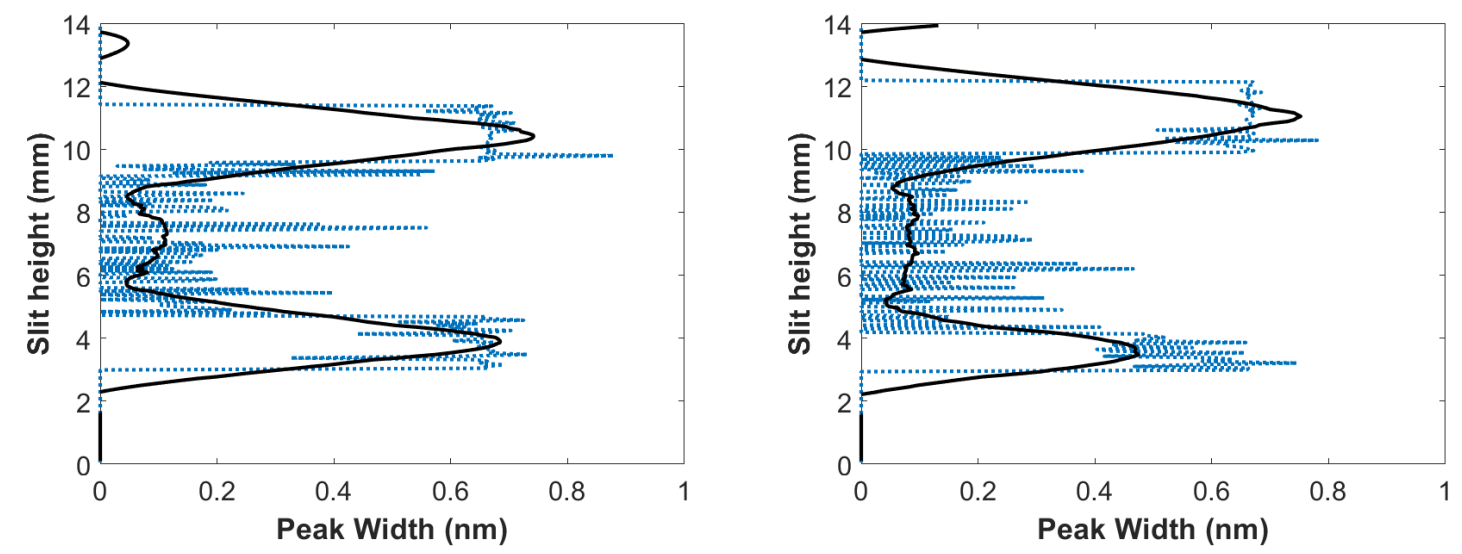

Figure 10. CN flowing-gas inferred widths of C I $193.09 \mathrm{~nm}$ atomic carbon line in second order vs. slit height for time delays of (left) 700 and (right) $950 \mathrm{ns.}$ 
Using Equation (3), electron densities could be calculated for the flowing-gas mixture. Figure 11 displays the calculated electron densities versus slit height, and the electron densities were on the order of $\simeq 10^{17} \mathrm{~cm}^{-3}$ in the central region. Higher electron densities were seen toward the edges of the plasma, and smaller electron densities were near the center of plasma, analogous to the results for the fixed gas mixture. Additionally, the plasma at $450 \mathrm{~ns}$ delay in Figure 11 displayed higher electron densities near the shockwave (at $\approx 1.9 \mathrm{~mm}$ ), which is consistent with the Taylor-Sedov blast-wave model and the presented experiment results with a fixed gas mixture in the cell.
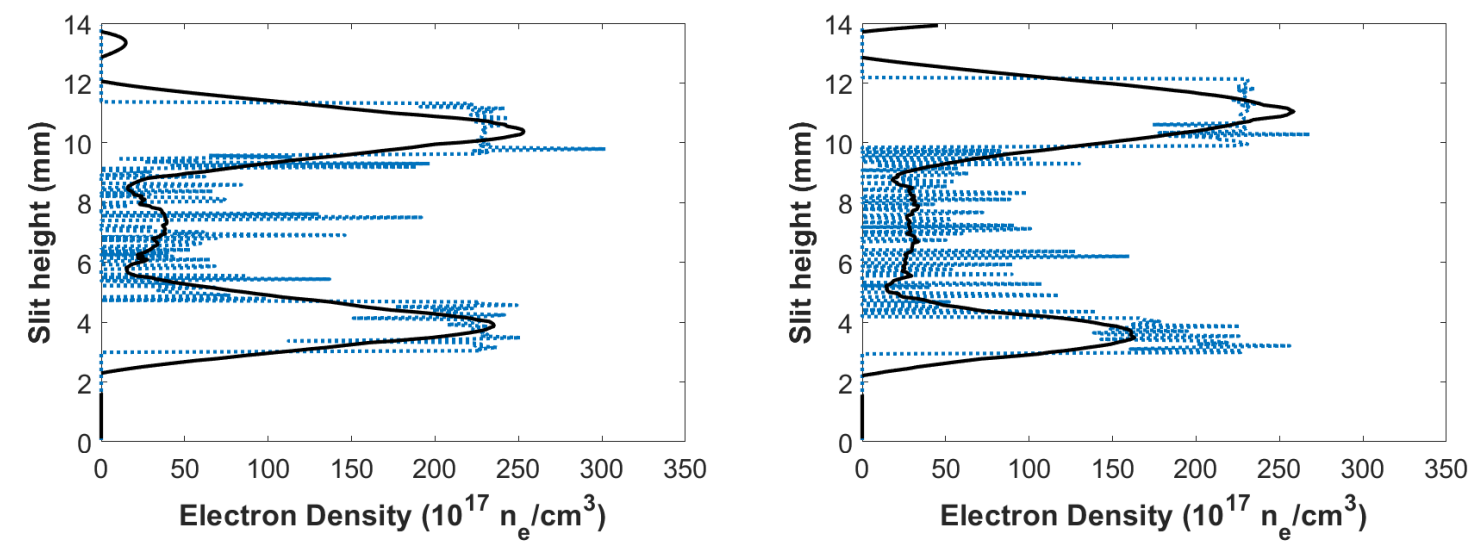

Figure 11. Calculated CN flowing-gas electron densities vs. slit height for time delays of (left) 450 and (right) $950 \mathrm{~ns}$.

\subsection{Abel-Inverted Spectra}

In view of the shadowgraphs that were recorded in the 0.5 to $1 \mu \mathrm{s}$ range (see Figures 1 and 2), it would be acceptable to apply an Abel inverse transform. Deviation from spherical symmetry is minimal for time delays of the order of $1 \mu$ s provided laser-induced excitation is accomplished with nanosecond IR radiation of the order of $200 \mathrm{~mJ} /$ pulse or less. Analysis of the molecular CN spectra follows the same symmetrization methods as applied for atomic hydrogen spectra [30,31].

The use of Chebyshev polynomials for Abel inversion of integral equation

$$
I(z, \lambda)=2 \int_{z}^{\rho} \varepsilon(r, \lambda) \frac{r}{\sqrt{r^{2}-z^{2}}} d r
$$

allows one to directly invert measured data. The $z$-direction corresponds to slit height, and line-of-sight integration was along the $y$-direction in view of Figure 1 . In this work, choosing the number of 15 polynomials for the inversion [32,33] maintained fidelity of the spectra and was equivalent to the use of a digital filter resulting in broadening computed radial spectra. A smaller number of polynomials would cause smaller spectral resolution. Measured line-of sight data $I(z, \lambda)$ along slit dimension $z$ were inverted for each wavelength $\lambda$ to obtain radial intensity distribution $\varepsilon(r, \lambda)$, with the upper limit much larger ( $\rho \gg R_{\text {plasma }}$ ) than radius, $R_{\text {plasma }}$ ) of the plasma.

The radial spectra were computed from the two-dimensional line-of-sight data (see Figures 3 and 4) that were accumulated with an intensified camera attached to the spectrometer, allowing one to resolve spectra along the slit. The recorded data were calibrated, corrected for system sensitivity using standard lamps, and prepared for Abel inversion. Usually, when recording 2D spectra, slight wavelength variations towards the edges of the slit occur, but these variations were taken care of in preparing the data for Abel inversion. Figures 12 and 13 illustrate the computed Abel inverted spectra. Only subtle differences are recognizable near the 2-2 CN band edge due to the $\mathrm{CI}$ line when comparing Figures 12 and 13; however, Figure 12 also contains electron-density information that confirmed higher electron density near the edges than the central regions, and, consequently, higher electron temperature for adiabatic expansion. 
(a)

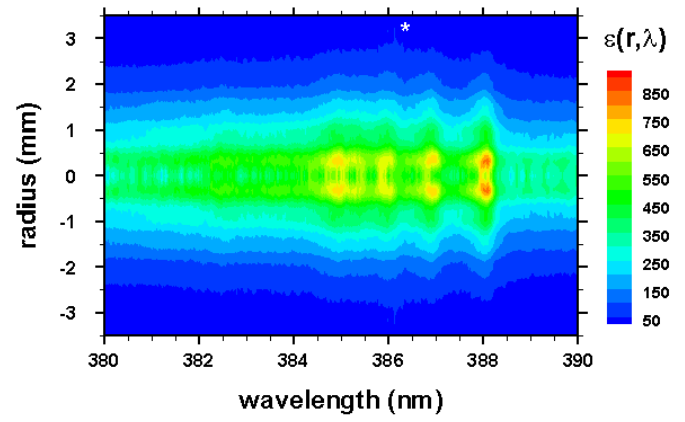

(b)

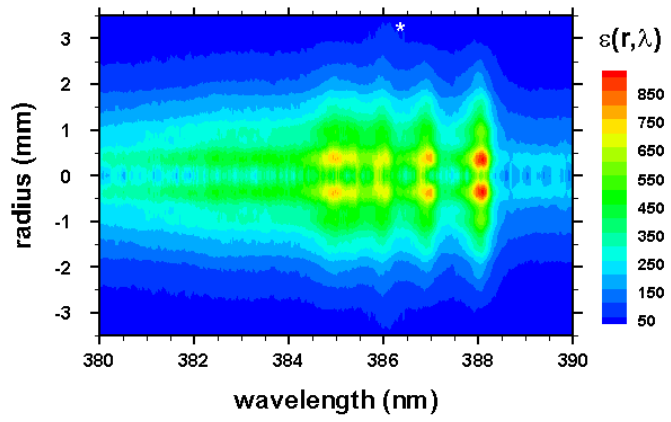

Figure 12. Abel-inverted data of Figure 3 for time delays of (a) 450 and (b) 950 ns. * , second-order neutral carbon line.

(a)

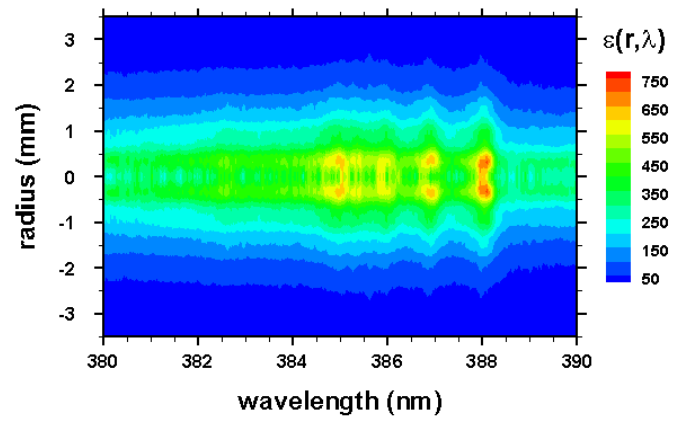

(b)

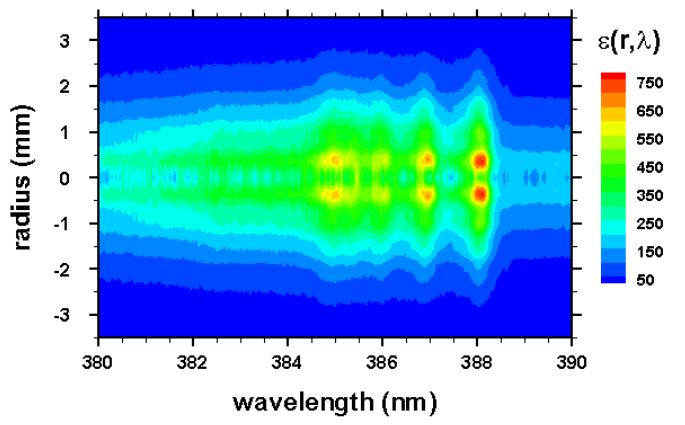

Figure 13. Abel-inverted data of Figure 4 for time delays of (a) 450 and (b) 950 ns.

Analysis of Abel-inverted data was expected to reveal similar results for the shockwave, but in view of the shadowgraphs in Figures 1 and 2, variations of $\mathrm{CN}$ distribution inside the shockwave and the plasma kernel were anticipated.

\section{Conclusions}

The main results of the work are summarized here: Primarily shockwave expansion affects the formation of $\mathrm{CN}$ molecules as the plasma expands. For time delays of the order of $1 \mu \mathrm{s}$, higher $\mathrm{CN}$ and electron concentrations occur near the shockwave than those in the central region of the plasma. The novelty of the laser-plasma experiments includes measurements of shockwave-expansion characteristics by tracing molecular $\mathrm{CN}$ formed by recombination. For time delays of the order of several $\mu$ s after plasma generation, regular $\mathrm{CN}$ molecular distributions occur in the plasma, namely, hotter central than cooler peripheral plasma, as indicated by $\mathrm{CN}$ signals.

Laser-plasma expansion following optical breakdown occurs at well above hypersonic speed. Measurements of recombination $\mathrm{CN}$ spectra within the first few microseconds yielded results as expected from atomic hydrogen Balmer series laser spectroscopy: Analysis revealed higher electron and higher $\mathrm{CN}$ excitation temperature near the shockwave than in the central region for time delays of the order of one microsecond. The expansion characteristics were deduced from systematic analysis of the recorded line-of-sight spectra. Application of Abel inversion was reasonable and supported by shadowgraphs recorded in SATP air for laser-plasma initiation for similar energy/pulse, as utilized for measurements of molecular spectra.

Further development of $\mathrm{CN}$ diagnosis with laser spectroscopy should focus on accurate measurements of spatial distributions with simultaneous recording of spectra at different angles. This would allow the examination of deviation from spherical symmetry by invoking inverse Radon transforms, viz. by applying computed tomography. For the various applications mentioned in this work, measurements of spatial distribution as plasma expands would also alleviate averaging along the line of sight. 
Author Contributions: All authors contributed equally to this work, and have read and agreed to the published version of the manuscript.

Funding: This research received no external funding.

Acknowledgments: Three of the authors (CGP, CMH, BSJ) wish to acknowledge the support in part by the Center for Laser Applications at the University of Tennessee Space Institute.

Conflicts of Interest: The authors declare no conflict of interest.

\section{References}

1. Parigger, C.G.; Helstern, C.M.; Gautam, G. Molecular Emission Spectroscopy of Cyanide in Laser-induced Plasma. Int. Rev. At. Mol. Phys. 2017, 8, 25-35.

2. Fent, K.W.; Evans, D.E.; Babik, K.; Striley, C.; Bertke, S.; Kerber, S.; Smith, D.; Horn, G.P. Airborne contaminants during controlled residential fires. J. Occup. Environ. Hyg. 2018, 15, 399-412. [CrossRef] [PubMed]

3. Arslanov, D.D.; Castro, M.P.P.; Creemers, N.A.; Neerincx, A.H.; Spunei, M.; Mandon, J.; Cristescu, S.M.; Merkus, P.; Harren, F.J.M. Optical parametric oscillator-based photoacoustic detection of hydrogen cyanide for biomedical applications. J. Biomed. Opt. 2013, 18, 107002. [CrossRef] [PubMed]

4. Ambrose, J.L.; Zhou, Y.; Haase, K.; Mayne, H.R.; Talbot, R.; Sive, B.C. A gas chromatographic instrument for measurement of hydrogen cyanide in the lower atmosphere. Atmos. Meas. Tech. 2012, 5, 1229-1240. [CrossRef]

5. Mudder, T.I.; Botz, M.M. Cyanide and society: A critical review. Eur. J. Miner. Process. Environ. Protect. 2004, 4, 62-74.

6. Bolstad-Johnson, D.M.; Burgess, J.L.; Crutchfield, C.D.; Storment, S.; Gerkin, R.; Wilson, J.R. Characterization of firefighter exposures during fire overhaul. Am. Ind. Hyg. Assoc. 2000, 61, 636-641. [CrossRef]

7. Betke, T.; Rommelmann, P.; Oike, K.; Asano, Y.; Groger, H. Cyanide-Free and Broadly Applicable Enantioselective Synthetic Platform for Chiral Nitriles through a Biocatalytic Approach. Angew. Chem. Int. Edit. 2017, 56, 12361-12366. [CrossRef]

8. Metzner, R.; Okazaki, S.; Asano, Y.; Groger, H. Cyanide-free Enantioselective Synthesis of Nitriles: Synthetic Proof of a Biocatalytic Concept and Mechanistic Insights. Chem. Cat. Chem 2014, 6, 3105-3109. [CrossRef]

9. Plass, C.; Hinzmann, A.; Terhorst, M.; Brauer, W.; Oike, K.; Yavuzer, H.; Asano, Y.; Vorholt, A.; Betke, T.; Gröger, H. Approaching Bulk Chemical Nitriles from Alkenes: A Hydrogen Cyanide-Free Approach through a Combination of Hydroformylation and Biocatalysis. Am. Chem. Soc. Catal. 2019, 9, 5198-5203. [CrossRef]

10. Hilson, G.; Monhemius, A.J. Alternatives to cyanide in the gold mining industry: What prospects for the future. J. Clean. Prod. 2006, 14, 1158-1167. [CrossRef]

11. Karlsson, H.L. Ammonia, nitrous oxide and hydrogen cyanide emissions from five passenger vehicles. Sci. Total Environ. 2004, 334-335, 125-132. [CrossRef]

12. Mekuto, L.; Ntwampe, S.K.O.; Akcil, A. An integrated biological approach for treatment of cyanidation wastewater. Sci. Total Environ. 2014, 571,711-720. [CrossRef]

13. Akcil, A.; Erust, C.; Gahan,C.S.; Ozgun, M.; Sahin, M.; Tuncuk, A. Precious metal recovery from waste printed circuit boards using cyanide and non-cyanide lixiviants-A review. Waste Manag. 2015, 45, 258-271. [CrossRef] [PubMed]

14. Álvarez, R.; Ordóñez, A.; Loredo, J.; Younger, P.L. Wetland-based passive treatment systems for gold ore processing effluents containing residual cyanide, metals and nitrogen species. Environ. Sci. Process. Impacts 2013, 15, 2115-2124. [CrossRef] [PubMed]

15. Moussa, S.G.; Leithead, A.; Li, S.-M.; Chan, T.W.; Wentzell, J.J.B.; Stroud, C.; Zhang, J.; Lee, P.; Jeffery, G.L.; Brook, R.; et al. Emissions of hydrogen cyanide from on-road gasoline and diesel vehicles. Atmos. Environ. 2016, 131, 185-195. [CrossRef] [PubMed]

16. Baum, M.M.; Moss, J.A.; Pastel, S.H.; Poskrebyshev, G.A. Hydrogen cyanide exhaust emissions from in-use motor vehicles. Environ. Sci. Technol. 2007, 41, 857-862. [CrossRef] [PubMed]

17. Merten, J.; Jones, M.; Hoke, S.; Allen, S. Differential Spectral Imaging of the CN Violet Band in Laser-Induced Plasmas on TNT Simulant Molecules. Phys. Conf. Ser. 2014, 548, 012042. [CrossRef]

18. Dagaut, P.; Glarborg, P.; Alzueta, M.U. The oxidation of hydrogen cyanide and related chemistry. Prog. Energy Combust. 2008, 34, 1-46. [CrossRef] [PubMed] 
19. Cremers, D.A.; Radziemski, L.J. Handbook of Laser-Induced Breakdown Spectroscopy; John Wiley \& Sons Ltd.: Hoboken, NJ, USA, 2006. [CrossRef]

20. Gautam, G.; Helstern, C.M.; Drake, K.A.; Parigger, C.G. Imaging of Laser-induced Plasma Expansion Dynamics in Ambient Air. Int. Rev. At. Mol. Phys. 2016, 7, 45-51. [CrossRef]

21. Taylor, G.I. The formation of a blast wave by a very intense explosion.-II. The atomic explosion of 1945 . Proc. Roy. Soc. A 1950 201, 175-186.

22. Thiyagarajan, M.; Thompson, S. Optical breakdown threshold investigation of $1064 \mathrm{~nm}$ laser induced air plasmas. J. Appl. Phys. 2012, 111, 073302.

23. Kogelnik, H.; Li, T. Laser Beams and Resonators. Appl. Opt. 1966, 5, 1550-1567.

24. Technical Note: Gaussian Beam Optics. Available online: https://www.newport.com/n/gaussian-beamoptics (accessed on 11 January 2020). [CrossRef]

25. Nelder, J.A.; Mead, R. A simplex method for function minimization. Comput. J. 1965, 7, 308-313. [CrossRef] [PubMed]

26. Parigger, C.G.; Woods, A.C.; Surmick, D.M.; Gautam, G.; Witte, M.J.; Hornkohl, J.O. Computation of diatomic molecular spectra for selected transitions of aluminum monoxide, cyanide, diatomic carbon, and titanium monoxide. Spectrochim. Acta Part B At. Spectrosc. 2015, 107, 132-138.

27. Parigger, C.G.; Hornkohl, J.O. Quantum Mechanics of the Diatomic Molecule with Applications; IOP Publishing: Bristol, UK, 2019. [CrossRef]

28. Dackman, M. Laser-Induced Breakdown Spectroscopy for Analysis of High-Density Methane-Oxygen Mixtures. Master's Thesis, University of Tennessee, Knoxville, TN, USA, 2014. [CrossRef]

29. Griem, H.R. Spectral Line Broadening by Plasmas; Academic Press: New York, NY, USA, 1974.

30. Parigger, C.G.; Gautam, G.; Surmick, D.M. Radial electron density measurements in laser-induced plasma from Abel inverted hydrogen Balmer beta line profiles. Int. Rev. At. Mol. Phys. 2015,6, 43-55.

31. Parigger, C.G.; Surmick, D.M.; Gautam, G. Self-absorption characteristics of measured laser-induced plasma line shapes. Phys. Conf. Ser. 2017, 810, 012012.

32. Pretzler, G. A New Method for Numerical Abel-Inversion. Z. Naturforsch. 1991, 46a, 639-641.

33. Pretzler, G.; Jäger, H.; Neger, T.; Philipp, H.; Woisetschläger, J. Comparison of Different Methods of Abel Inversion Using Computer Simulated and Experimental Side-On Data. Z. Naturforsch. 1992, 47a, 955-970. [CrossRef]

Sample Availability: Not available.

(C) 2020 by the authors. Licensee MDPI, Basel, Switzerland. This article is an open access article distributed under the terms and conditions of the Creative Commons Attribution (CC BY) license (http:/ / creativecommons.org/licenses/by/4.0/). 